\title{
Periaqueductal Grey Stimulation Induced Panic-Like Behaviour Is Accompanied by Deactivation of the Deep Cerebellar Nuclei
}

\author{
Véronique M. P. Moers-Hornikx • Johan S. H. Vles • Lee Wei Lim • Mustafa Ayyildiz • \\ Sủleyman Kaplan • Antonio W. D. Gavilanes • Govert Hoogland • \\ Harry W. M. Steinbusch • Yasin Temel
}

Published online: 16 November 2010

(C) The Author(s) 2010. This article is published with open access at Springerlink.com

\begin{abstract}
Until recently, the cerebellum was primarily considered to be a structure involved in motor behaviour. New anatomical and clinical evidence has shown that the cerebellum is also involved in higher cognitive functions and non-motor behavioural changes. Functional imaging in patients with anxiety disorders and in cholecystokinin tetrapeptide-induced panic-attacks shows activation changes in the cerebellum. Deep brain stimulation of the dorsolateral periaqueductal grey (dlPAG) and the ventromedial hypothalamus (VMH) in rats has been shown to induce escape behaviour, which mimics a panic attack in humans. We used this animal model to study the neuronal activation in the deep cerebellar nuclei (DCbN) using c-Fos immunohistochemistry. c-Fos expression in the $\mathrm{DCbN}$ decreased significantly after inducing escape behaviour by stimulation of the dIPAG and the VMH, indicating that the DCbN were deactivated. This study demonstrates that the
\end{abstract}

V. M. P. Moers-Hornikx · L. W. Lim · G. Hoogland ·

H. W. M. Steinbusch • Y. Temel

Department of Neuroscience, Maastricht University,

P.O. Box 616, 6200 MD Maastricht, The Netherlands

V. M. P. Moers-Hornikx $(\varangle) \cdot$ J. S. H. Vles

Department of Neurology, Maastricht University Medical Centre,

P.O. Box 5800, 6202 AZ Maastricht, The Netherlands

e-mail: v.moers@mumc.nl

L. W. Lim · G. Hoogland · Y. Temel

Department of Neurosurgery,

Maastricht University Medical Centre,

P.O. Box 5800, 6202 AZ Maastricht, The Netherlands

\section{S. Kaplan}

Department of Histology \& Embryology,

Medical School of Ondokuz Mayis University,

Samsun, Turkey
$\mathrm{DCbN}$ are directly or indirectly involved in panic attacks. We suggest that the cerebellum plays a role in the selection of relevant information, and that deactivation of the cerebellar nuclei is required to allow inappropriate behaviour to occur, such as panic attacks.

Keywords Cerebellum · Deep brain stimulation . Periaqueductal grey - Ventromedial hypothalamus . Escape behaviour
Abbreviations
CCAS Cerebellar cognitive affective syndrome
OCD Obsessive-compulsive disorder
CCK-4 Cholecystokinin tetrapeptide
PAG Periaqueductal grey
DBS Deep brain stimulation
dIPAG Dorsolateral periaqueductal grey

M. Ayyildiz

Department of Physiology,

Medical School of Ondokuz Mayis University,

Samsun, Turkey

V. M. P. Moers-Hornikx • L. W. Lim • G. Hoogland •

H. W. M. Steinbusch · Y. Temel

European Graduate School of Neuroscience (EURON),

Maastricht, The Netherlands

\author{
A. W. D. Gavilanes \\ Department of Pediatrics-Neonatology, \\ Maastricht University Medical Centre, \\ P.O. Box 5800, 6202 AZ Maastricht, The Netherlands
}




$\begin{array}{ll}\text { VMH } & \text { Ventromedial hypothalamus } \\ \text { DCbN } & \text { Deep cerebellar nuclei } \\ \text { OF } & \text { Open field } \\ \text { FN } & \text { Fastigial nucleus of the cerebellum } \\ \text { DN } & \text { Dentate nucleus of the cerebellum } \\ \text { IN } & \text { Interposite nucleus of the cerebellum } \\ \text { c-Fos-ir } & \text { c-Fos immunoreactive } \\ \text { PC } & \text { Purkinje cell }\end{array}$

\section{Introduction}

The cerebellum has long been considered to be predominantly involved in motor coordination and control $[1,2]$. The last two decades, however, it has become increasingly clear that the cerebellum is also involved in behaviour and cognition $[3,4]$. Schmahmann and Sherman described the cerebellar cognitive affective syndrome consisting of impairment of executive functions, difficulties with spatial cognition, changes of personality including disinhibited or inappropriate behaviour and language deficits [5]. Recent neuroanatomical and functional imaging research has pointed towards a role for the cerebellum in neuropsychiatric diseases such as autism, schizophrenia and mood and anxiety disorders [611]. With respect to anxiety disorders, Levinson described the existence of cerebellar-vestibular dysfunction in $94 \%$ of patients with various types of anxiety disorders [12]. De Bellis and Kuchibhatla showed that children and adolescents with post-traumatic stress disorder related to maltreatment have significantly smaller cerebelli than paediatric subjects with generalized anxiety disorder and healthy subjects [13]. Several groups have shown structural and functional differences in the cerebellum of patients with anxiety disorders compared to control subjects [14-22]. In patients with obsessive-compulsive disorder (OCD), a disorder characterised by anxiety, an increase in grey matter was found in the anterior cerebellum [22]. Using functional imaging, Busatto et al. found an increased activation in the superior cerebellum of OCD patients compared to control subjects [14]. Another group showed that after treatment, OCD patients showed a decreased activation in the cerebellum compared to pretreatment scans [15]. In contrast, Nabeyama et al. found decreased activation in untreated OCD patients compared to control subjects, which increased after successful treatment of these patients [16]. In patients with panic disorder, an increased activation of the cerebellum was found when compared to control patients [20]; this activation decreased again after successful treatment of these patients [21]. In healthy individuals in whom panic attacks were induced by cholecystokinin tetrapeptide (CCK-4), an increased activation of the cerebellum was found, especially in the vermis [23-26]. However, the significance of these findings is not yet clear.
Panic disorder is one of the most frequently encountered anxiety disorders [27]. Panic attacks consist of an acute, sometimes unexpected onset of intense fear, accompanied by a variety of physical symptoms [27]. Treatment usually includes behavioural and pharmacological therapies, either alone or in combination. It is thought that panic attacks may be the result of an abnormally sensitive fear network [28]. The periaqueductal grey (PAG) is known to be part of this fear network which further consists of the central nucleus of the amygdala, ventromedial hypothalamus (VMH), hippocampus and other brainstem regions [28]. Deep brain stimulation (DBS) in rats of the dorsolateral periaqueductal grey (dPAG) and one of its target structures, the VMH has been shown to elicit a typical behaviour consisting of vigorous running and jumping [29]. This behaviour, also known as escape behaviour, is accompanied by strong emotional and autonomic activation, and thereby mimics panic attacks in humans [29, 30].

In the present study, we used DBS of the dIPAG and the VMH as a model of panic attack to evaluate the involvement of the deep cerebellar nuclei $(\mathrm{DCbN})$. In a previous study, we have found that increased impulsivity in rats was accompanied by a deactivation of the DCbN [4]. These findings suggested that the cerebellum plays a role in regulating appropriate behaviour. Based on their findings in a patient with pathological laughter and crying, Parvizi et al. hypothesized that the cerebellum adjusts behavioural responses to the correct situational context of a stimulus, and that when the cerebellum operates on the basis of incorrect information, this could lead to inappropriate or even chaotic behaviour [31]. We hypothesized that deactivation of the $\mathrm{DCbN}$ was needed for pathological behaviour to occur. In line with this, here we predicted to find again a deactivation of the $\mathrm{DCbN}$ in rats with panic-like behaviour. Panic attacks consist of an inadequate response to a stimulus. Possibly the cerebellum also plays a role in regulation of the response to these stimuli by selection of relevant information. Deactivation of the $\mathrm{DCbN}$ may then be linked to an inadequate response to a certain stimulus by disabling this selection process. To analyze activation patterns, we have used c-Fos immunohistochemistry. We focused on the $\mathrm{DCbN}$ as these are the output structures of the cerebellum. c-Fos was chosen as it is an immediate early gene which is considered to reflect neuronal activation [32, 33]. The level of c-Fos protein is maximal about $2 \mathrm{~h}$ after administration of a stimulus and disappears again after 4 to $8 \mathrm{~h}$ even if the stimulus is continued [32]. Therefore, the c-Fos expression found $2 \mathrm{~h}$ after a specific stimulus in controlled conditions can be considered to result directly from the stimulus, and this expression can be used to identify brain areas influenced by this stimulus [33]. 


\section{Materials and Methods}

\section{Animals}

The animals used were male albino Wistar rats $(n=15$, 12 weeks old, bred and housed at the Central Animal Facility of the Maastricht University, the Netherlands), with an average body weight of 300-350 g at the time of surgery. Rats were housed individually in standard cages with sawdust bedding in an air-conditioned room under a 12/12-h reversed light/dark cycle. Food, standard laboratory chow (Hopefarms, Woerden, the Netherlands) and water were available ad libitum. All experiments were approved by the Animal Experiments and Ethics Committee of Maastricht University.

\section{Surgical Procedure}

Rats were randomly assigned to one of the following three experimental groups: control (no surgery), dIPAG DBS or VMH DBS. A detailed description of the surgical procedure was reported previously [29, 34]. In brief, animals were anaesthetized throughout the entire procedure using a combination of ketamine $(90 \mathrm{mg} / \mathrm{kg})$ and xylazine $(10 \mathrm{mg} / \mathrm{kg})$ injected subcutaneously. Rats were placed in a stereotactic apparatus (Stoelting, Wood Dale, IL, USA; model 51653). After making a burr hole, rats received implantation of a unilateral electrode at the level of the right dIPAG (coordinates from Bregma, $\mathrm{AP}=-7.6 \mathrm{~mm}, \mathrm{ML}=$ $0.7 \mathrm{~mm}$ and $\mathrm{V}=-4.8 \mathrm{~mm}$; approached with a coronal angle of $10^{\circ}$ ) and the right $\mathrm{VMH}$ (coordinates from Bregma, $\mathrm{AP}=-2.5 \mathrm{~mm}, \mathrm{ML}=0.5 \mathrm{~mm}$ and $\mathrm{V}=-9.5 \mathrm{~mm}$ ). A construction of one electrode with an inner wire of a platinum-irridium combination (Technomed, Beek, the Netherlands), with a tip diameter of $50 \mu \mathrm{m}$ and a shaft diameter of $250 \mu \mathrm{m}$, was implanted in this experiment [35]. The electrodes were fixed in position using dental cement.

\section{Deep Brain Stimulation}

After a recovery period of about 1 week, all animals underwent a first stimulation session to determine the escape threshold. The stimulation amplitudes were gradually increased until escape behaviour was observed. At each step, stimulation duration was $15 \mathrm{~s}$ followed by a period without stimulation of $45 \mathrm{~s}$. The stimulation frequency was set at $50 \mathrm{~Hz}$ and pulse width at $0.1 \mathrm{~ms}$ based on previous experiments [29]. A World Precision Instruments digital stimulator (DS8000, WPI, Berlin, Germany) and a stimulus isolator (DLS100, WPI, Berlin, Germany) were used to deliver the stimuli. Real-time verification of the parameters applied during stimulation was obtained using a digital oscilloscope (Agilent 54622D oscilloscope, Agilent Technologies, Amstelveen, the Netherlands). After confirming the current amplitudes necessary to evoke escape behaviour, all rats had a period of 2 weeks without stimulation before the final stimulation session in the open field (OF) arena was performed. Control rats were handled and placed in the OF similarly.

\section{Behavioural Evaluation}

Rats were evaluated in an OF test. For more details of this test, please see Lim et al. [29]. In summary, rats were placed in the arena and were connected with an external stimulator through externalized leads. The stimulation started approximately $1 \mathrm{~min}$ after the rat was placed in the OF arena, using the current amplitudes previously eliciting escape behaviour. The behaviour of the rats was videotaped (Ethovision, Noldus Information Technology, Wageningen, the Netherlands).

\section{Histological Processing}

Two hours after the final stimulation session, rats were deeply anesthetized and perfused transcardially with Tyrode $(0.1 \mathrm{M})$, followed by a fixative containing $4 \%$ paraformaldehyde, $15 \%$ picric acid and $0.05 \%$ gluteraldehyde in $0.1 \mathrm{M}$ phosphate buffer ( $\mathrm{pH}$ 7.6). The brains were removed and postfixed for $2 \mathrm{~h}$ followed by immersion in $20 \%$ sucrose at $4^{\circ} \mathrm{C}$ until saturated. Brain tissue was then quickly frozen with $\mathrm{CO} 2$ and stored at $-80^{\circ} \mathrm{C}$. The cerebellum was cut serially into $10-\mu \mathrm{m}$ sections, which were collected on gelatine-coated glasses. We used a previously published protocol for c-Fos immunohistochemistry [4]. In summary, this staining was carried out by incubating sections two nights with a polyclonal rabbit anti-c-Fos antibody (Santa Cruz Biotechnology Inc., Santa Cruz, CA, USA).This was followed by overnight incubation with the secondary antibody (biotinylated donkey anti-rabbit, Jackson Immunoresearch Laboratories Inc., Westgrove, PA, USA). Subsequently, the sections were incubated with an avidin-biotin-peroxidase complex (Elite ABC-kit, Vectastatin; Burlingame, CA, USA) for $2 \mathrm{~h}$. To visualize the immune complex of horseradish peroxide reaction product, sections were incubated with 3,3'-diaminobenzidine tetrahydrochloride (DAB)/nickel chloride (NiCl2) solution for $10 \mathrm{~min}$. After dehydrating, all sections were cover-slipped with Pertex (HistolabProducts ab, Goteborg, Sweden). Additionally, for each animal, the area of the dIPAG and VMH was cut into $30-\mu \mathrm{m}$ sections and stained with standard haematoxylineosin (Merck, Darmstadt, Germany) staining to locate the electrode tips.

Stereological Quantification of c-Fos Immunoreactive Cells

Stereological analysis was performed with a stereology workstation (CAST-GRID-Computer Assisted Stereological 
Toolbox-Olympus, Denmark). After exactly tracing boundaries of the fastigial nucleus (FN) and dentate nucleus of the cerebellum (DN) on microscopic video images displayed on a monitor, numbers of c-Fos immunoreactive (c-Fos-ir) cells in both nuclei were evaluated with the modified Abercrombie cell counting method [36]. All neurons of which the cell bodies gave a positive reaction within an unbiased counting frame distributed in a systematic-random fashion throughout the delineated regions were counted. Estimated numbers of neurons were calculated from the numbers of counted neurons and the corresponding sampling probability. The numerical density $\left(N_{\mathrm{V}}\right)$ of c-Fos-ir cells in the nuclei was estimated using the formula $N_{\mathrm{V}}=$ $\left(N_{\mathrm{A}} / T\right) \cdot(T /(T+D))$ [36]. In this formula, $N_{\mathrm{A}}, T$ and $D$ are the positive cell number in the unbiased counting frame, the section thickness and the mean particle diameter, respectively. To make an acceptable biological comment on any subject, one should estimate total number of cells rather than numerical density of cells in the interested region. For this reason, we estimated the volume $(V)$ of each cerebellar nucleus by multiplying the total area of each nucleus that is seen in the whole section of cerebellum $(\Sigma A)$ with the mean section thickness $(T)$, as shown in this formula $V=\sum A \cdot T$ [37]. The total number of cells in a nucleus was estimated by the formula $N=N_{\mathrm{V}} \cdot V$.

\section{Semiquantative Analysis of c-Fos-Immunoreactive Cells}

Analysis of the c-Fos-ir cells in the interposite nucleus of the cerebellum (IN) was performed using a previously described semiquantitative method [4]. In summary, photographs of the IN were taken at $\times 4$ magnification using an Olympus DP70 camera connected to an Olympus AX70 bright-field microscope (analySIS; Imaging System,
Münster, Germany). The same light intensity and threshold settings of the camera were employed for all sections. For each animal, we selected two sections of the IN for quantification. The boundaries of the IN were delineated and the area measured. The counting of the c-Fos-ir cells was performed using the image analysis program 'Image J' (version 1.38, NIH, USA). A cell was counted as c-Fos-ir if its density was $65 \%$ higher than the mean background density of that section. Artefacts in the sections were excluded from analysis to ensure the accuracy of measurements. The number of c-Fos-ir cells per square millimetre was calculated.

\section{Statistical Analysis}

The data of the c-Fos counts were analysed using the Kruskal-Wallis test for non-parametric data. Nonparametric testing was chosen due to small group sizes. A value of $p<0.05$ was considered significant. Post hoc analysis was performed using the Mann-Whitney test. Since we expected a deactivation in the stimulated groups, we used a one-tailed procedure.

\section{Results}

Histological Evaluation of the Electrode Localisation

In all animals included in this study, the electrode tips were positioned correctly in the dIPAG and $\mathrm{VMH}$, respectively (Fig. 1). No histological damage was observed except for the electrode trajectory with the standard haematoxylineosin staining, suggesting that stimulation with the current stimulation settings did not cause observable tissue damage.

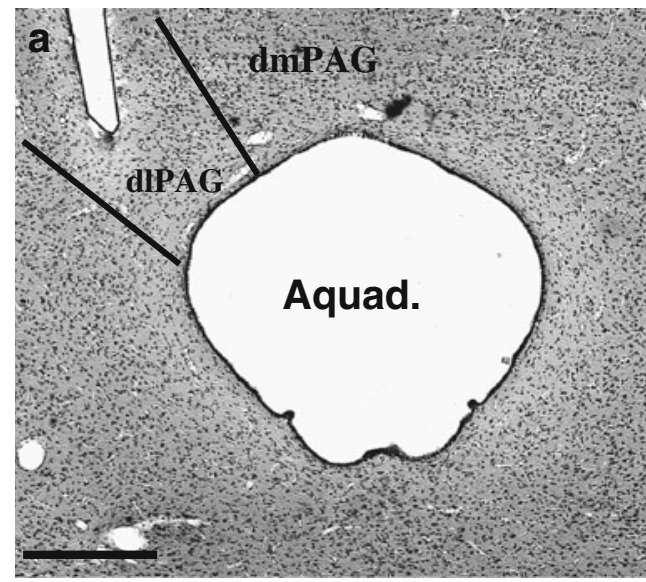

Fig. 1 Representative low-power photomicrographs of 30- $\mu$ m-thick frontal sections from the brain of a rat subjected to stereotactic implantation of a concentric bipolar electrode to stimulate the dIPAG (a, scale bar $=250 \mu \mathrm{m})$ and VMH $(\mathbf{b}$, scale bar $=500 \mu \mathrm{m})$. The tips of

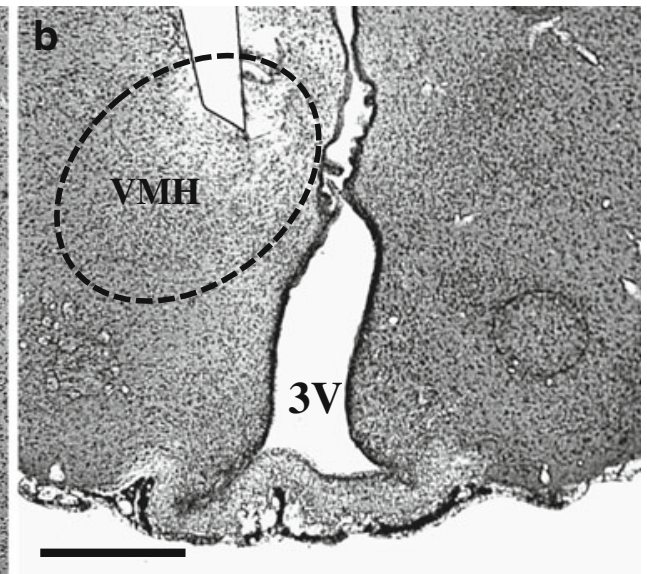

the electrodes are situated within the respective targets. Aquad aqueduct of Sylvius, $d m P A G$ dorsomedial periaqueductal grey, dlPAG dorsolateral periaqueductal grey, $3 \mathrm{~V}$ third ventricle, $V M H$ ventromedial hypothalamus 


\section{Behavioural Evaluation}

The intensity of the electrical current applied to the dIPAG and VMH of the animals was based on the threshold shown to induce escape behaviour. All rats showed 'escape behaviour' characterized by rigorous and aimless running. The current amplitudes necessary for inducing escape behaviour were significantly different between rats with dlPAG- and VMH-DBS $(F \mathrm{~s}>351.13 ; p<0.00)$. The mean current density applied to the dIPAG to induce escape behaviour was approximately $90 \mu \mathrm{A}$ and for the $\mathrm{VMH}$, approximately $600 \mu \mathrm{A}$.

Evaluation of the Number of c-Fos-Immunoreactive Cells

There was a significant decrease in the amount of c-Fos-ir cells in the dentate nucleus $\left(H_{2}=6.343, p<0.05\right.$; Figs. 2 and $3)$ and in the fastigial nucleus $\left(H_{2}=6.870, p<0.05\right.$; Figs. 2 and 3) after DBS. As expected, we found a lower amount of c-Fos-ir cells in the dentate and fastigial nucleus of the stimulated rats compared to the control rats. Post hoc analysis showed that in the rats stimulated in the dlPAG, the difference with control rats was significant for both nuclei (DN, $U=1.00, r=-2.21$; FN, $U=0.00, r=-2.45$ ). When comparing VMH-stimulated rats to control rats, this difference was significant only in the nucleus dentatus $(U=1.00, r=-2.02)$; the difference in the fastigial nucleus showed a trend towards significance $(U=2.00, r=-1.73)$.

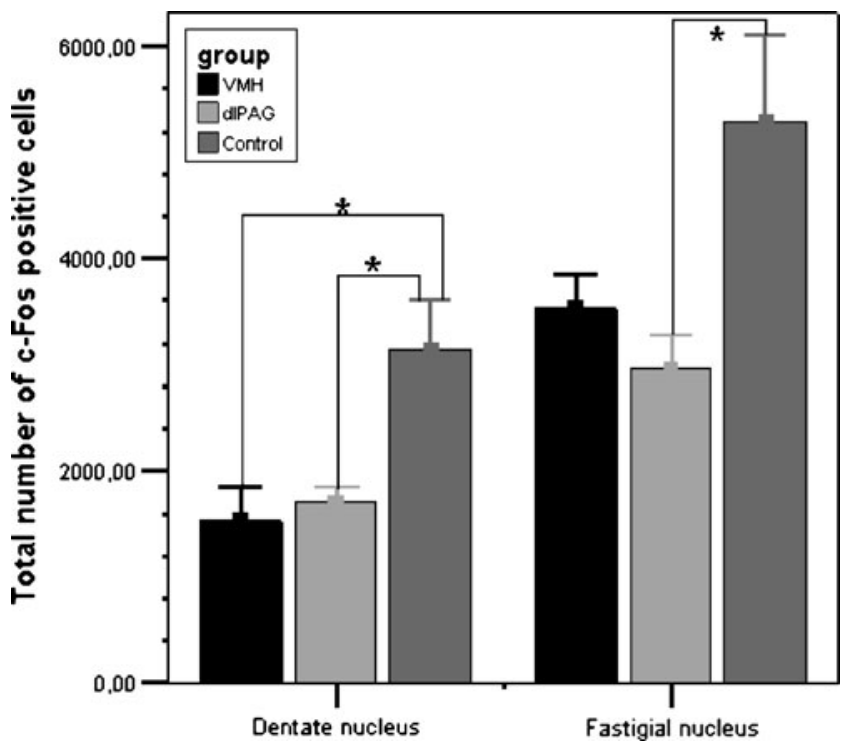

Fig. 2 c-Fos expression in the dentate nucleus and in the fastigial nucleus of the cerebellum. Data are represented as means plus SEM showing control animals, animals stimulated in the dorsolateral periaqueductal grey (dlPAG) and animals stimulated in the ventromedial hypothalamus (VMH). The asterisk indicates a significant difference between groups. Note the significantly lower numbers of c-Fos immunoreactive neurons in the groups with animals showing panic attacks evoked by stimulation of the dIPAG and VMH
There was no significant difference in the amount of c-Fos-ir cells between the stimulated groups ( $\mathrm{DN}, U=7.00, r=-0.74$; FN, $U=6.00, r=-0.98)$.

Analysis of the interposite nucleus (IN) showed a similar trend towards decreased c-Fos expression in the stimulated rats compared to controls (PAG, $U=19.00, r=-1.04$; VMH, $U=21.00, r=-1.37)$. Again, there was no difference between the stimulated groups $(U=36.00, r=-0.36)$.

\section{Discussion}

The objective of our experiment was to use electrical stimulation of the dIPAG and of the VMH in rats as a model of panic attack to study the involvement of the DCbN. We have found that panic-like behaviour was accompanied by a decrease of c-Fos-ir cells in the DCbN, indicating deactivation. c-Fos expression was significantly lower in the DN of both treatment groups and in the FN of the dIPAG DBS group when compared to controls. In the VMH DBS group, c-Fos expression in the FN was lower compared to controls, with a trend towards significance. Using a semiquantitative analysis, the IN showed a similar trend towards decreased c-Fos expression in the stimulated rats compared to controls. This should be considered a preliminary finding since the method of counting was not performed using stereological principles.

There is anatomical and functional evidence supporting the role of the cerebellum in panic. Sakai et al. found a significantly higher glucose uptake in the cerebellum of patients with panic disorder compared to control patients [20]. After clinical improvement of these patients due to cognitive-behavioural therapy, the glucose uptake in cerebellum had decreased [21]. Several groups found increased activation of the cerebellum after CCK-4-induced panic attacks in healthy subjects [24, 25]. This increase was not seen in these subjects during anticipatory anxiety. These studies were not initially designed to analyse changes in the cerebellum, and the changes found are usually unexpected. The findings are often simply mentioned as interesting or surprising, although several groups relate to the role of the cerebellum in fear conditioning as proposed by Sacchetti et al. [38]. Sacchetti et al. reviewed the evidence showing that the cerebellar vermis plays a role in the fear response and in fear conditioning, especially in fear consolidation. The most important changes are thought to take place at the level of the Purkinje cell (PC). It has been shown that several forms of fear conditioning lead to increased PC excitability and an increased firing rate of the PCs. In contrast, heterozygous Lurcher mice, which show early and complete apoptosis of cerebellar PCs, show reduced inhibition to anxietyprovoking aversive areas [39]. In summary, it seems that increased PC activity leads to more fear, and decreased or 

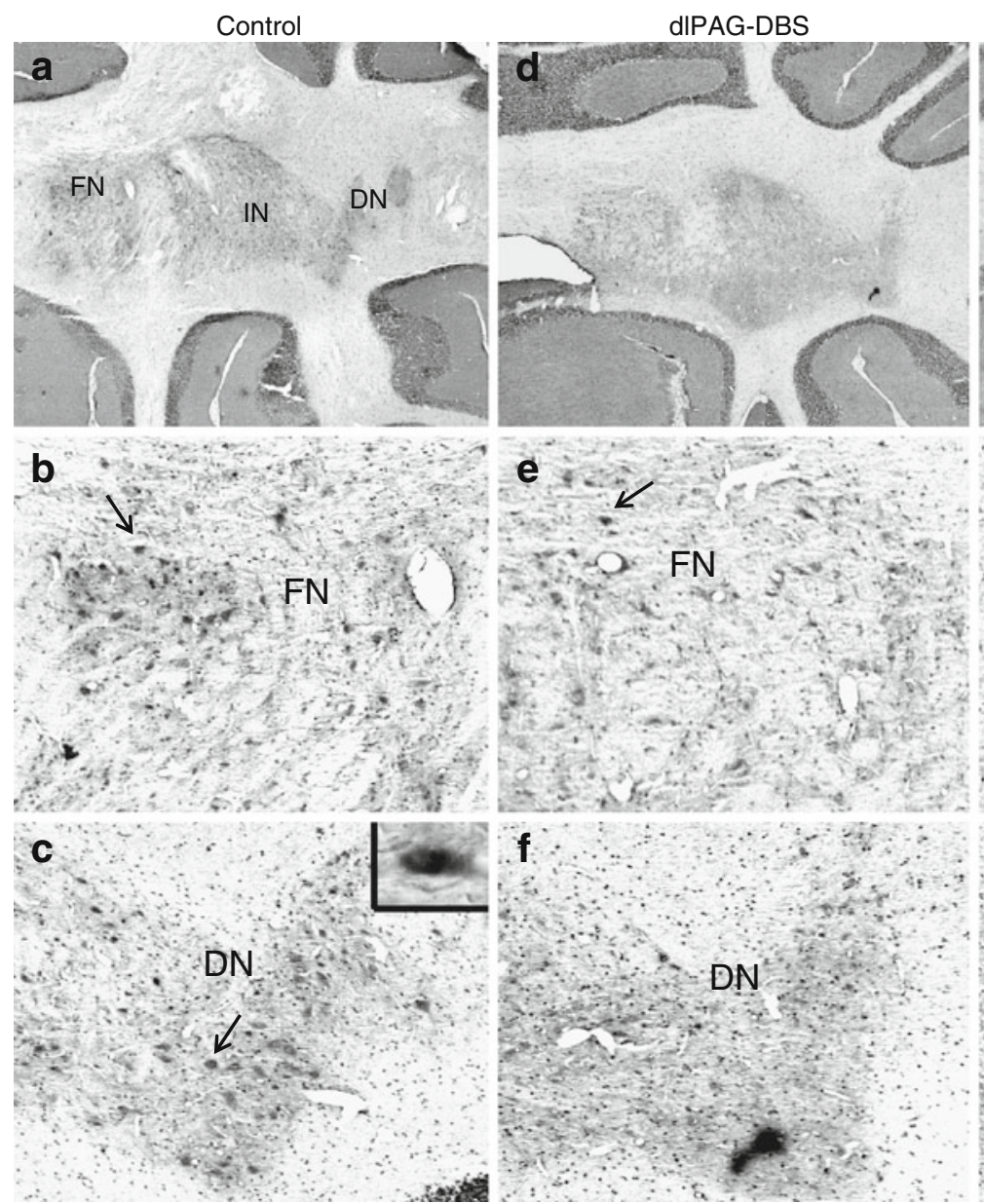

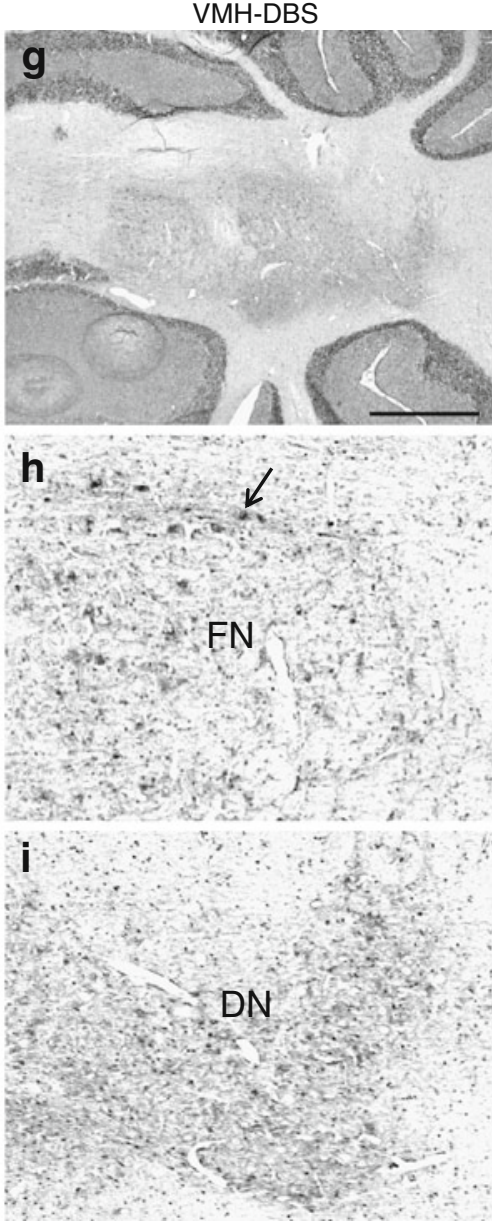

Fig. 3 Representative low-power photomicrographs of c-Fos expression in a $10-\mu \mathrm{m}$-thick horizontal section of the deep cerebellar nuclei at Bregma level $-6.10 \mathrm{~mm}$ (according to the stereotactic rat brain atlas of Paxinos and Watson of 1998) in a sham rat (a-c), a rat stimulated in the dIPAG $(\mathbf{d}-\mathbf{f})$, and a rat stimulated in the VMH $(\mathbf{g}-\mathbf{i})$. Panels $\mathbf{a}, \mathbf{d}$, and $\mathbf{g}$ were taken at $\times 2$ magnification, and $\mathbf{b}, \mathbf{c}, \mathbf{e}, \mathbf{f}, \mathbf{h}$, and $\mathbf{i}$ were taken at high-power magnification. The arrows point to a c-Fos-ir cell, represented by a small dark dot. The inset in $\mathbf{c}$ shows a representative high-power photomicrograph of a c-Fos-ir cell. Scale bar for $\times 2$ magnification power $=1 \mathrm{~mm}$. $d I P A G$ dorsolateral periaqueductal grey, $V M H$ ventromedial hypothalamus, $D B S$ deep brain stimulation, $D N$ dentate nucleus of the cerebellum, $F N$ fastigial nucleus of the cerebellum absent PC activity to less fear. Functional imaging is designed to analyse activation changes in the cerebral cortex; therefore, it is likely that the activation seen in the cerebellum is also located in the cortex. Increased activation found in functional imaging studies may therefore reflect the increased activity of the PCs. Since PCs are known to have an inhibitory action on the $\mathrm{DCbN}$, these findings are in line with deactivation that we found in the $\mathrm{DCbN}$ in the stimulated rats which show panic-like behaviour.

In the cerebellum, the vermis, projecting through the fastigial nucleus, seems to be the most important structure in fear and panic. In the cases described by Schmahmann et al., the vermis was always involved in patients with changes of affect [5]. In early experiments, vermal lesions were shown to attenuate a variety of fear behaviour, whereas vermal stimulation leads to increased fear-related responses [40, 41] Other animal research shows a selective role for the fastigial nucleus in heart-rate conditioning [40, $42,43]$. The vermis also contributes to consolidation of fear memory [44]. There is also evidence of a role of the interposite nucleus and the dentate nucleus in fear. In studies investigating several aspects of fear conditioning, animals with lesions of the dentate and interposite nucleus do not acquire an aversive conditioned response but acquire an appetitive conditioned response [45] and show unaltered heart-rate conditioning [42] and vocalisation indicative of unspecific fear [46]. Furthermore, there are clear bidirectional connections to the hypothalamus from (greatest to least concentration) the dentate nucleus, the interposite nucleus and the fastigial nucleus [47-50], supporting a role of all $\mathrm{DCbN}$ in autonomic processes, for example, those 
related to fear. In addition, the $\mathrm{DCbN}$ have been shown to project to several parts of the fear network: Teune et al. injected tracers in the $\mathrm{DCbN}$ in rats and documented projections to the PAG from the fastigial nucleus and the dentate nucleus and, in lesser degree, from the interposite nucleus [51]. Several groups have suggested projections from the fastigial nucleus to the amygdala, hippocampus, septal nuclei and the nucleus accumbens, based on behavioural changes following cerebellar stimulation or lesioning [38, 41, 52]. Berntson and Torello, for example, showed that hyperemotionality caused by septal lesions was largely attenuated by lesions of the fastigial nucleus [53]. An exact anatomical pathway has never been shown [54]. However, these behavioural changes indicate that the involvement of the cerebellum in fear does not merely consist of regulation of an autonomic visceromotor response but that there is a place for the cerebellum in the network regulating fear processing [38]. In a recent review, Stoodley and Schmahmann present a functional somatotopy of the non-motor functions of the cerebellum based on functional imaging [55]. They conclude that the vermis of the posterior lobe seems to be specifically related to emotional processing, whereas activation in the posterior cerebellar hemispheres may be related to the decisionmaking aspects of the tasks used in the experimental setting [55]. On the other hand, Timman et al. reviewed anatomical evidence for a role of the cerebellum in emotional and cognitive learning, and they conclude that with respect to fear, the vermis, projecting through the fastigial nucleus, contributes to the autonomic and somatic aspects, whereas the posterolateral cerebellar hemispheres, projecting through the dentate and interposite nucleus, play a role in the emotional content of fear processing [40].

In summary, there is ample evidence that all cerebellar nuclei are involved in fear, in which the fastigial nucleus possibly mediates a different aspect of fear than the dentate nucleus and the interposite nucleus. This correlates with our findings in the present study showing a similar deactivation in the dentate nucleus and in the fastigial nucleus, and possibly also in the interposite nucleus. Increased fear is associated with increased PC activity in functional imaging studies, which is hypothesised to lead to inhibition of the $\mathrm{DCbN}$ and therefore of cerebellar output. We speculate that the cerebellum plays a role in regulating appropriate behaviour in response to any stimulus, and that a decreased cerebellar output may play a role in emergence of an inappropriate response, such as a panic attack. This decreased output may be in response to incorrect input, as suggested by Parvizi et al. [31]; however, we suggest that the cerebellum also plays a direct role in the selection of relevant information on which an adequate behavioural response is based, and that deactivation of the $\mathrm{DCbN}$ then leads to inappropriate behaviour by inhibiting this selection process.

\section{Conclusion}

This study supports the hypothesis that the cerebellum is involved in panic attacks. Using DBS of the dIPAG and the VMH in rats as a model of a panic attack, we found that the activity of the $\mathrm{DCbN}$ as measured by c-Fos expression decreased significantly in the dentate nucleus and in the fastigial nucleus, and possibly also in the interposite nucleus. In a previous study, we showed that the $\mathrm{DCbN}$ are deactivated in rats showing increased impulsivity. In conclusion, deactivation of the cerebellum is associated with inappropriate behaviour such as panic and impulsivity. We suggest that the cerebellum plays a role in the selection of relevant information, thereby inhibiting such inappropriate behaviour.

Acknowledgements The scientific work of Y. Temel has received funding from the Netherlands Organisation of Scientific Research (NWO-Veni grant) and the Dutch Brain Foundation (Hersenstichting Nederland). The authors are grateful to Mrs. Hellen Steinbusch for her technical assistance.

Conflicts of interest The authors of this manuscript withhold no conflicts of interest.

Open Access This article is distributed under the terms of the Creative Commons Attribution Noncommercial License which permits any noncommercial use, distribution, and reproduction in any medium, provided the original author(s) and source are credited.

\section{References}

1. Fine EJ, Ionita CC, Lohr L. The history of the development of the cerebellar examination. Semin Neurol. 2002;22(4):375-84.

2. Schmahmann JD. Disorders of the cerebellum: ataxia, dysmetria of thought, and the cerebellar cognitive affective syndrome. J Neuropsychiatry Clin Neurosci. 2004;16(3):367-78.

3. Leiner HC, Leiner AL, Dow RS. Does the cerebellum contribute to mental skills? Behav Neurosci. 1986;100(4):443-54.

4. Moers-Hornikx VM, Sesia T, Basar K, Lim LW, Hoogland G, Steinbusch HW, et al. Cerebellar nuclei are involved in impulsive behaviour. Behav Brain Res. 2009;203(2):256-63.

5. Schmahmann JD, Sherman JC. The cerebellar cognitive affective syndrome. Brain. 1998;121:561-79.

6. Baldacara L, Borgio JG, de Lacerda AL, Jackowski AP. Cerebellum and psychiatric disorders. Rev Bras Psiquiatr. 2008;30(3):281-9.

7. Hoppenbrouwers SS, Schutter DJ, Fitzgerald PB, Chen R, Daskalakis ZJ. The role of the cerebellum in the pathophysiology and treatment of neuropsychiatric disorders: a review. Brain Res Rev. 2008;59(1):185-200.

8. Andreasen NC, Pierson R. The role of the cerebellum in schizophrenia. Biol Psychiatry. 2008;64(2):81-8.

9. Picard H, Amado I, Mouchet-Mages S, Olie JP, Krebs MO. The role of the cerebellum in schizophrenia: an update of clinical, cognitive, and functional evidences. Schizophr Bull. 2008;34(1):155-72.

10. Palmen SJ, van Engeland H, Hof PR, Schmitz C. Neuropathological findings in autism. Brain. 2004;127(Pt 12):2572-83.

11. Pierce K, Courchesne E. Evidence for a cerebellar role in reduced exploration and stereotyped behavior in autism. Biol Psychiatry. 2001;49(8):655-64. 
12. Levinson HN. The cerebellar-vestibular predisposition to anxiety disorders. Percept Mot Skills. 1989;68(1):323-38.

13. De Bellis MD, Kuchibhatla M. Cerebellar volumes in pediatric maltreatment-related posttraumatic stress disorder. Biol Psychiatry. 2006;60(7):697-703.

14. Busatto GF, Zamignani DR, Buchpiguel CA, Garrido GE, Glabus $\mathrm{MF}$, Rocha ET, et al. A voxel-based investigation of regional cerebral blood flow abnormalities in obsessive-compulsive disorder using single photon emission computed tomography (SPECT). Psychiatry Res. 2000;99(1):15-27.

15. Kang DH, Kwon JS, Kim JJ, Youn T, Park HJ, Kim MS, et al. Brain glucose metabolic changes associated with neuropsychological improvements after 4 months of treatment in patients with obsessive-compulsive disorder. Acta Psychiatr Scand. 2003;107 (4):291-7.

16. Nabeyama M, Nakagawa A, Yoshiura T, Nakao T, Nakatani E, Togao $\mathrm{O}$, et al. Functional MRI study of brain activation alterations in patients with obsessive-compulsive disorder after symptom improvement. Psychiatry Res. 2008;163(3):236-47.

17. Kilts CD, Kelsey JE, Knight B, Ely TD, Bowman FD, Gross RE, et al. The neural correlates of social anxiety disorder and response to pharmacotherapy. Neuropsychopharmacology. 2006;31 (10):2243-53

18. Tillfors M, Furmark T, Marteinsdottir I, Fredrikson M. Cerebral blood flow during anticipation of public speaking in social phobia: a PET study. Biol Psychiatry. 2002;52(11):1113-9.

19. Warwick JM, Carey P, Jordaan GP, Dupont P, Stein DJ. Resting brain perfusion in social anxiety disorder: a voxel-wise whole brain comparison with healthy control subjects. Prog Neuropsychopharmacol Biol Psychiatry. 2008;32(5):1251-6.

20. Sakai Y, Kumano H, Nishikawa M, Sakano Y, Kaiya H, Imabayashi E, et al. Cerebral glucose metabolism associated with a fear network in panic disorder. NeuroReport. 2005;16(9):92731.

21. Sakai Y, Kumano H, Nishikawa M, Sakano Y, Kaiya H, Imabayashi E, et al. Changes in cerebral glucose utilization in patients with panic disorder treated with cognitive-behavioral therapy. Neuroimage. 2006;33(1):218-26.

22. Pujol J, Soriano-Mas C, Alonso P, Cardoner N, Menchon JM, Deus J, et al. Mapping structural brain alterations in obsessive-compulsive disorder. Arch Gen Psychiatry. 2004;61(7): 720-30.

23. Javanmard M, Shlik J, Kennedy SH, Vaccarino FJ, Houle S, Bradwejn J. Neuroanatomic correlates of CCK-4-induced panic attacks in healthy humans: a comparison of two time points. Biol Psychiatry. 1999;45(7):872-82.

24. Eser D, Leicht G, Lutz J, Wenninger S, Kirsch V, Schule C, et al. Functional neuroanatomy of CCK-4-induced panic attacks in healthy volunteers. Hum Brain Mapp. 2007;30(2):511-22.

25. Schunck T, Erb G, Mathis A, Gilles C, Namer IJ, Hode Y, et al. Functional magnetic resonance imaging characterization of CCK4-induced panic attack and subsequent anticipatory anxiety. Neuroimage. 2006;31(3):1197-208.

26. Benkelfat C, Bradwejn J, Meyer E, Ellenbogen M, Milot S, Gjedde A, et al. Functional neuroanatomy of CCK4-induced anxiety in normal healthy volunteers. Am J Psychiatry. 1995;152 (8): $1180-4$.

27. Roy-Byrne PP, Craske MG, Stein MB. Panic disorder. Lancet. 2006;368(9540):1023-32.

28. Gorman JM, Kent JM, Sullivan GM, Coplan JD. Neuroanatomical hypothesis of panic disorder, revised. Am J Psychiatry. 2000;157 (4):493-505.

29. Lim LW, Blokland A, Visser-Vandewalle V, Vlamings R, Sesia T, Steinbusch $\mathrm{H}$, et al. High-frequency stimulation of the dorsolateral periaqueductal gray and ventromedial hypothalamus fails to inhibit panic-like behaviour. Behav Brain Res. 2008;193(2):197203.

30. Schenberg LC, Bittencourt AS, Sudre EC, Vargas LC. Modeling panic attacks. Neurosci Biobehav Rev. 2001;25(78):647-59.

31. Parvizi J, Anderson SW, Martin CO, Damasio H, Damasio AR. Pathological laughter and crying: a link to the cerebellum. Brain. 2001;124(Pt 9):1708-19.

32. Sharp FR, Sagar SM, Hicks K, Lowenstein D, Hisanaga K. c-fos mRNA, Fos, and Fos-related antigen induction by hypertonic saline and stress. J Neurosci. 1991;11(8):2321-31.

33. Smith MA, Banerjee S, Gold PW, Glowa J. Induction of c-fos mRNA in rat brain by conditioned and unconditioned stressors. Brain Res. 1992;578(1-2):135-41.

34. Temel Y, Boothman LJ, Blokland A, Magill PJ, Steinbusch HW, Visser-Vandewalle V, et al. Inhibition of 5-HT neuron activity and induction of depressive-like behavior by high-frequency stimulation of the subthalamic nucleus. Proc Natl Acad Sci USA. 2007;104(43):17087-92.

35. Temel Y, Visser-Vandewalle V, van der Wolf M, Spincemaille GH, Desbonnet L, Hoogland G, et al. Monopolar versus bipolar high frequency stimulation in the rat subthalamic nucleus: differences in histological damage. Neurosci Lett. 2004;367(1):92-6.

36. Abercrombie M. Estimation of nuclear population from microtome sections. Anat Rec. 1946;94:239-47.

37. Tunc AT, Turgut M, Aslan H, Sahin B, Yurtseven ME, Kaplan S. Neonatal pinealectomy induces Purkinje cell loss in the cerebellum of the chick: a stereological study. Brain Res. 2006;1067(1): 95-102.

38. Sacchetti B, Scelfo B, Strata P. The cerebellum: synaptic changes and fear conditioning. Neuroscientist. 2005;11:217-27.

39. Lorivel T, Hilber P. Effects of chlordiazepoxide on the emotional reactivity and motor capacities in the cerebellar Lurcher mutant mice. Behav Brain Res. 2006;173(1):122-8.

40. Timmann D, Drepper J, Frings M, Maschke M, Richter S, Gerwig M, et al. The human cerebellum contributes to motor, emotional and cognitive associative learning. A review. Cortex. 2010;46(7): 845-57.

41. Snider RS, Maiti A. Cerebellar contributions to the Papez circuit. J Neurosci Res. 1976;2(2):133-46.

42. Lavond DG, Lincoln JS, McCormick DA, Thompson RF. Effect of bilateral lesions of the dentate and interpositus cerebellar nuclei on conditioning of heart-rate and nictitating membrane/eyelid responses in the rabbit. Brain Res. 1984;305(2): 323-30.

43. Supple Jr WF, Leaton RN. Lesions of the cerebellar vermis and cerebellar hemispheres: effects on heart rate conditioning in rats. Behav Neurosci. 1990;104(6):934-47.

44. Sacchetti B, Scelfo B, Tempia F, Strata P. Long-term synaptic changes induced in the cerebellar cortex by fear conditioning. Neuron. 2004;42:973-82.

45. Steinmetz JE, Logue SF, Miller DP. Using signaled barpressing tasks to study the neural substrates of appetitive and aversive learning in rats: behavioral manipulations and cerebellar lesions. Behav Neurosci. 1993;107(6):941-54.

46. Lee T, Kim JJ. Differential effects of cerebellar, amygdalar, and hippocampal lesions on classical eyeblink conditioning in rats. J Neurosci. 2004;24(13):3242-50.

47. Haines DE, Dietrichs E, Mihailoff GA, McDonald EF. The cerebellar-hypothalamic axis: basic circuits and clinical observations. Int Rev Neurobiol. 1997;41:83-107.

48. Zhu JN, Zhang YP, Song YN, Wang JJ. Cerebellar interpositus nuclear and gastric vagal afferent inputs reach and converge onto 
glycemia-sensitive neurons of the ventromedial hypothalamic nucleus in rats. Neurosci Res. 2004;48:405-17.

49. Çavdar S, San T, Aker R, Sehirli ÜS, Onat FYI. Cerebellar connections to the dorsomedial and posterior nuclei of the hypothalamus in the rat. J Anat. 2001;198:37-45.

50. Dietrichs E, Haines DE. Possible pathways for cerebellar modulation of autonomic responses: micturition. Scand J Urol Nephrol Suppl. 2002;210:16-20.

51. Teune TM, Voogd J, Ruigrok TJH. Topography of cerebellar nuclear projections to the brain stem in the rat. Prog Brain Res. 2000;124:141-72.
52. Turner BM, Paradiso S, Marvel CL, Pierson R, Boles Ponto LL, Hichwa RD, et al. The cerebellum and emotional experience. Neuropsychologia. 2007;45:1331-41.

53. Berntson GG, Torello MW. Attenuation of septal hyperemotionality by cerebellar fastigial lesions in the rat. Physiol Behav. 1980;24(3):547-51.

54. Strick PL, Dum RP, Fiez JA. Cerebellum and nonmotor function. Annu Rev Neurosci. 2009;32:413-34.

55. Stoodley CJ, Schmahmann JD. Functional topography in the human cerebellum: a meta-analysis of neuroimaging studies. Neuroimage. 2009;44(2):489-501. 